\title{
Anti-inflammatory effects of empagliflozin in patients with type 2 diabetes and insulin resistance
}

\author{
Sachiko Hattori* (D)
}

\begin{abstract}
Background: Inflammation might be a pathological mediator of cardiovascular events in patients with type 2 diabetes and high cardiovascular risk.

Methods: We investigated whether empagliflozin (EMPA) exerts anti-inflammatory effects that are reflected in decreased high-sensitivity C-reactive protein (hsCRP) values. Patients were allocated to receive a placebo $(n=51)$ or EMPA ( $n=51)$ as an add-on treatment. Fasting blood samples were collected before and every 3 months after this intervention for 1 year.

Results: Empagliflozin tended to elicit reductions in $\mathrm{BMI}, \mathrm{HbA1c}$, aspartate aminotransferase, alanine aminotransferase (ALT), and gamma-glutamyl transpeptidase compared with the placebo, but the differences did not reach statistical significance. Levels of LDL-cholesterol, HDL-cholesterol, and triglycerides were unaltered, significantly increased, and decreased, respectively, by EMPA, but the differences were not statistically significant compared with the placebo. Empagliflozin for 12 months notably reduced the homeostatic model assessment of insulin resistance (HOMA-IR), remnant-like particle cholesterol (RLP-C), and hsCRP by $43 \%, 52 \%$ and $54 \%$, respectively. The time courses of these reductions significantly differed from those of the placebo. Systolic and diastolic blood pressure were also significantly reduced by EMPA compared with the placebo. We applied multiple linear regression analysis to determine which factors were associated with changes in hsCRP induced by EMPA. The results revealed that alterations in hsCRP values (log [hsCRP at 12 months] minus log [hsCRP at month 0]) were significantly associated with changes in HOMA-IR, RLP-C, systolic blood pressure, HDL-C and ALT.
\end{abstract}

Conclusion: Empagliflozin decreased hs-CRP and lowered levels of remnant related lipoproteins probably via ameliorating insulin resistance. The cardiovascular benefits conferred by EMPA might be driven at least partly by antiinflammatory effects, and this mechanism might cooperate with other EMPA-induced changes including reduced blood pressure, to achieve the degree of cardioprotection revealed by the EMPA-REG OUTCOME trial.

Trial registration UMIN Clinical Registry (UMIN000021552). Registered 21 March 2016, https://upload.umin.ac.jp/UMINO 00021552

Keywords: Sodium-glucose co-transporter-2 inhibitor, High sensitivity CRP, Insulin resistance

*Correspondence: sh07172017@olive.plala.or.jp

Department of Endocrinology and Metabolism, Tohto Clinic, 4-1,

Kioi-Cho, Chiyoda-Ku, Tokyo 102-0094, Japan 


\section{Background}

The sodium-glucose cotransporter 2 inhibitor, empagliflozin (EMPA) reduced relative risk for cardiovascular (CV) mortality, hospitalization for heart failure and death from any cause by $38 \%, 35 \%$ and $32 \%$, respectively, in the EMPA-REG OUTCOME trial of patients with type 2 diabetes and high risk of $\mathrm{CV}$ [1]. The results of a sub-analysis of the EMPA-REG OUTCOME study of Asian patients with type 2 diabetes and high $\mathrm{CV}$ risk were also striking [2]. Thus, the EMPA-REG OUTCOME trial established that EMPA is cardioprotective in high-risk patients with diabetes, but the underlying mechanisms remain elusive.

Obesity-related, subacute chronic inflammation is associated with incident type 2 diabetes and atherosclerotic CV disease. Inflammation might be a pathological mediator of these commonly concurrent pathologies. Ridker et al. [3] recently showed that antiinflammatory therapy with canakinumab, which targets the interleukin- $1 \beta$ innate immunity pathway, leads to a significantly lower rate of recurrent $\mathrm{CV}$ events than a placebo, independently of lowering LDL-cholesterol. They showed that the magnitude of the reduction in highsensitivity C-reactive protein (hsCRP) after a single dose of canakinumab might provide a simple clinical method with which to identify individuals who are likely to benefit from continued treatment; that is, lower is better for reducing inflammation with canakinumab [4].

Empagliflozin exerts anti-inflammatory effects in experimental animal models [5, 6], but not in humans. Here, we aimed to determine whether EMPA exerts anti-inflammatory effects and whether the $\mathrm{CV}$ benefits of EMPA are due partly to such effects, implied by decreased hsCRP values in patients with type 2 diabetes and insulin resistance.

\section{Methods}

\section{Study design and participants}

This single-center, open-label, randomized, prospective study enrolled patients without a history of medication with SGLT2 inhibitors and with HbA1c $>6.2 \%$ regardless of diet, exercise, and medical treatment other than SGLT2 inhibitors for at least 12 weeks. They were assessed at our clinic and might have had insulin resistance (BMI $>28$ or homeostatic model assessment of insulin resistance [HOMA-IR] $>1.73$ or fasting immunoreactive insulin [IRI] $>10$ and fasting blood glucose $[\mathrm{FBG}]<180)$. The patients were then allocated to receive EMPA $(10 \mathrm{mg} ; \mathrm{n}=51)$ or a placebo $(\mathrm{n}=51)$ as an add-on treatment. All patients continued with their administered oral hypoglycemic drugs (sulfonylureas, metformin, or an $\alpha$-glucosidase inhibitor), antihypertensive agents (angiotensin II receptor blockers or calcium channel blockers), and antihyperlipidemic agents (statins or fibrates) (Table 1).

\section{Measurements}

Overnight fasting blood and urine samples were obtained at baseline and after every 3 months of EMPA or placebo administration for 1 year. All biochemical data were obtained at our laboratory, except IRI, remnant-like particle cholesterol (RLP-C), hsCRP and urinary albumin, which were assessed at LSI Medicine Corporation (Tokyo, Japan). We calculated HOMA-IR every 3 months as follows: EMPA was stopped for $72 \mathrm{~h}$ until urinary glucose was undetectable and then blood values of fasting glucose and IRI were measured.

\section{Statistical analysis}

Data are expressed as mean \pm standard deviation (SD). Data were statistically analyzed using EZR software version 1.21 [7]. Parameters before and 3, 6, 9, and 12 months after treatment were compared using paired t-tests. Changes in parameters over a period of 12 months between placebo and EMPA were analyzed using repeated measures ANOVA. Factors associated with changes in hsCRP were determined using multiple linear regression analysis. Continuous and categorical baseline values and medications were analyzed using t-tests and Fisher exact tests, respectively. Differences were considered statistically significant at $\mathrm{p}<0.05$.

\begin{tabular}{llll}
$\begin{array}{l}\text { Table } 1 \text { Baseline } \\
\text { of the participants }\end{array}$ & charasteristics & and & medicatoins \\
\hline & Placebo $(\mathbf{n}=\mathbf{5 1})$ & EMPA $(\mathbf{n}=\mathbf{5 1})$ & P value \\
\hline Age (years) & $58.1 \pm 9.71$ & $57.4 \pm 12.3$ & 0.778 \\
Male/(female) & $41(10)$ & $38(13)$ & 0.636 \\
$\begin{array}{lll}\text { Baseline medication } \\
\text { Slfonylureas }\end{array}$ & 11 & 10 & \\
Metformin & 12 & 12 & 1 \\
a-Gl & 8 & 7 & 1 \\
ARB & 16 & 17 & 0.915 \\
CCB & 10 & 10 & 1 \\
Statins & 17 & 16 & 1 \\
Fibrates & 9 & 10 & 0.879
\end{tabular}

Data were expressed as mean \pm standard deviation

a-Gl a-glicosidase inhibitor, $A R B$ angiotensin II receptor blocker, $C C B$ calcium channel blocker 


\section{Results}

Levels of BMI, HbA1c, aspartate aminotransferase (AST), alanine aminotransferase (ALT) and gamma-glutamyl transpeptidase $(\gamma-$ GTP) were slightly but not significantly reduced by EMPA compared with the placebo. Levels of LDL-cholesterol, HDL-cholesterol, and triglycerides were unaltered, significantly increased, and reduced, respectively, by EMPA, but the differences did not reach statistical significance compared with the placebo. Notably, HOMA-IR, RLP-C, and hsCRP were reduced by 43\%, $52 \%$, and 54\%, respectively, after 12 months of EMPA. The time courses of these reductions significantly differed between EMPA and the placebo. Systolic (SBP) and diastolic blood pressure (DBP) were significantly reduced by EMPA, but not by the placebo. Estimated glomerular filtration rates (eGFR), urinary albumin excretion (measured as urinary albumin-to-creatinine ratios; ACR) did not significantly differ between the placebo and EMPA groups (Table 2, Fig. 1).

We applied multiple linear regression analysis to identify factors associated with changes in hsCRP in the EMPA group. The objective variable comprised changes in hsCRP over a period of 12 months (log [hsCRP at 12 months] minus log [hsCRP at month 0]), and changes in FBG, IRI, BMI, HbA1c, AST, ALT, $\gamma$-GTP, LDL-cholesterol, HDL-cholesterol, RLP-C, eGFR, SBP, and DBP over 12 months were included as explanatory variables. The findings of these analyses $\left(\mathrm{R}^{2}=0.9671, \mathrm{p}=4.709 \times 10^{-8}\right)$ revealed that changes in hsCRP were significantly associated with changes in HOMA-IR $(\mathrm{t}=2.541, \mathrm{p}=0.0274)$, RLP-C $\quad(t=2.528, \quad \mathrm{p}=0.0281), \quad$ SBP $\quad(t=-2.324$, $\mathrm{p}=0.0403)$, HDL-cholesterol $(\mathrm{t}=-2.411, \mathrm{p}=0.0346)$, and ALT $(\mathrm{t}=-2.372, \mathrm{p}=0.0370)$ (Table 3$)$.

\section{Discussion}

This study showed that the SGLT2 inhibitor, EMPA, decreases hsCRP, lowers levels of remnant lipoproteins and ameliorates insulin resistance.

Insulin resistance is not a simple matter of deficient glucose uptake in response to insulin, but a multifaceted syndrome that significantly increases risk for CV disease [8]. How SGLT2 inhibitors improve insulin resistance has been examined using a glucose clamp procedure [9, 10]. However, the observation periods were relatively short and the patient cohorts were quite small because long-term assessment using this technique is difficult to implement. Here, we repeatedly measured HOMA-IR to evaluate changes in insulin resistance. We found that EMPA decreased HOMA-IR and significantly decreased hsCRP and RLP-C.
Three reports have described an association between hsCRP and insulin resistance [11-13]. The present study found a significant association between decreased hsCRP and ameliorated HOMA-IR, suggesting that improved insulin resistance results in anti-inflammatory effects.

Remnant lipoproteins are thought to be atherogenic. Remnant-like particle cholesterol (RLP-C) reflects amounts of various remnant lipoproteins in the blood. Increased levels of RLP-C are thought to comprise a significant and independent risk factor for coronary artery disease (CAD) and to predict future coronary events in patients with CAD and type 2 diabetes [14]. The present study found a significant correlation between changes with EMPA in RLP-C and in hsCRP. We recently found that EMPA decreases RLP-C levels in close association with ameliorated insulin resistance at 3 months in patients with diabetes and insulin resistance [15]. The present study found that this suppressive effect of EMPA on RLP-C continued for 12 months.

The CV benefits of EMPA might be due in part to antiinflammatory effects as indicated by the $54 \%$ decrease in hsCRP in the present study. Arima et al. found that hsCRP levels were associated with future coronary heart disease events in a general Japanese population [16]. However, although hsCRP levels are far lower among Asians than other populations, the striking reduction in CV risk among Asians was similar to that in the overall population in the EMPA-REG OUTCOME trial [2]. Thus, a reduction in hsCRP within the lower range might confer a considerable benefit in $\mathrm{CV}$ risk reduction among Japanese patients with type 2 diabetes and insulin resistance.

This study has several limitations. We evaluated insulin resistance using HOMA-IR, which is not a good indicator under poor diabetes control. Another is that measuring HOMA-IR immediately after EMPA administration might not always reflect insulin resistance. This is because SGLT2 inhibitors work by inhibiting glucose reabsorption in the proximal tubules of the kidney, which results in increased urinary glucose excretion and decreased blood glucose levels. Thus, we excluded patients with poorly controlled diabetes from the present study. We also stopped EMPA administration for $72 \mathrm{~h}$ until SGLT2 inhibition became ineffective, then measured blood glucose and IRI in fasting blood samples to calculate HOMA-IR. Since the findings of linear regression analyses cannot define cause-and-effect between hSCRP and HOMA-IR and other parameters, further study is needed to clarify this issue. 


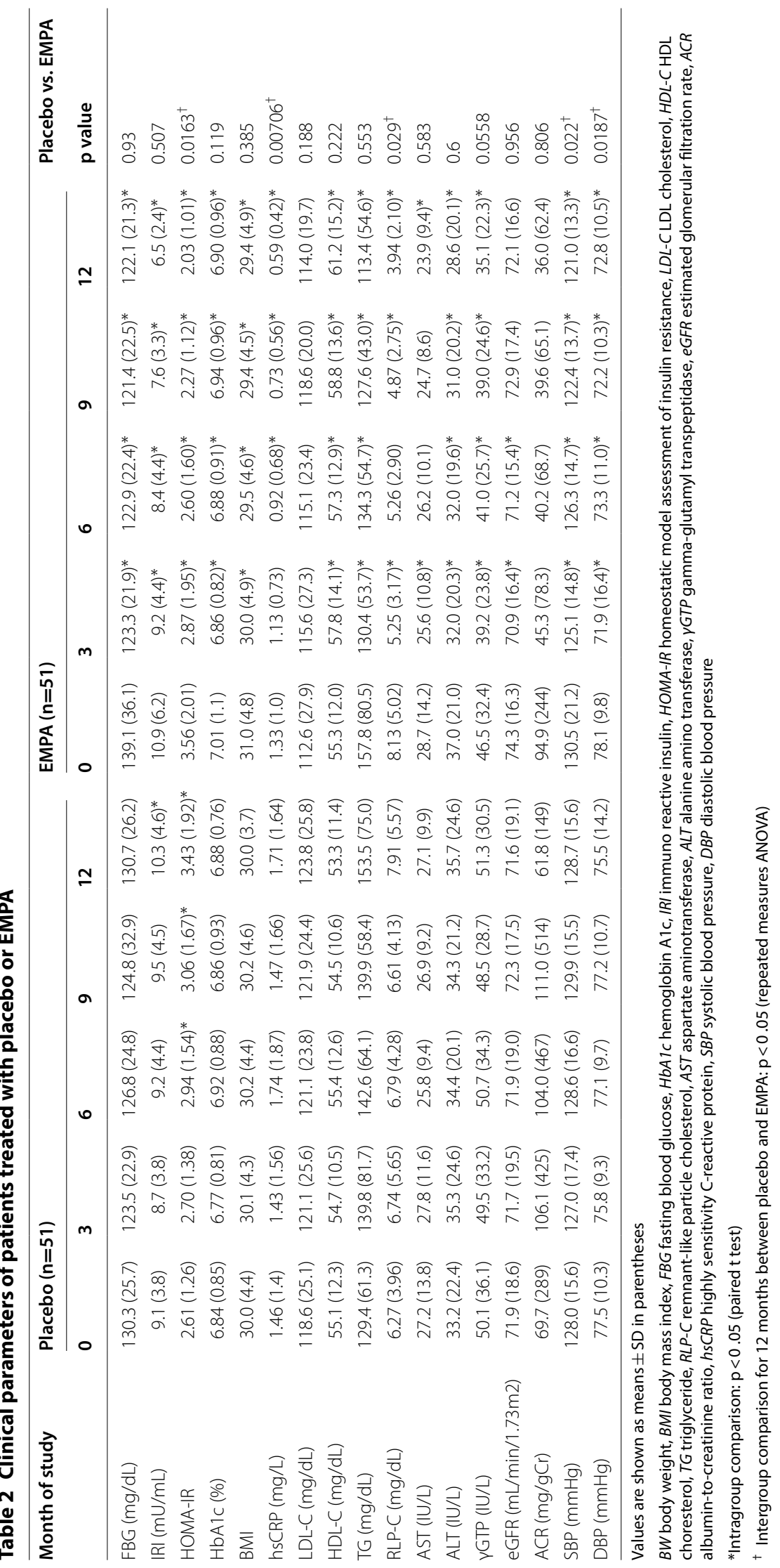



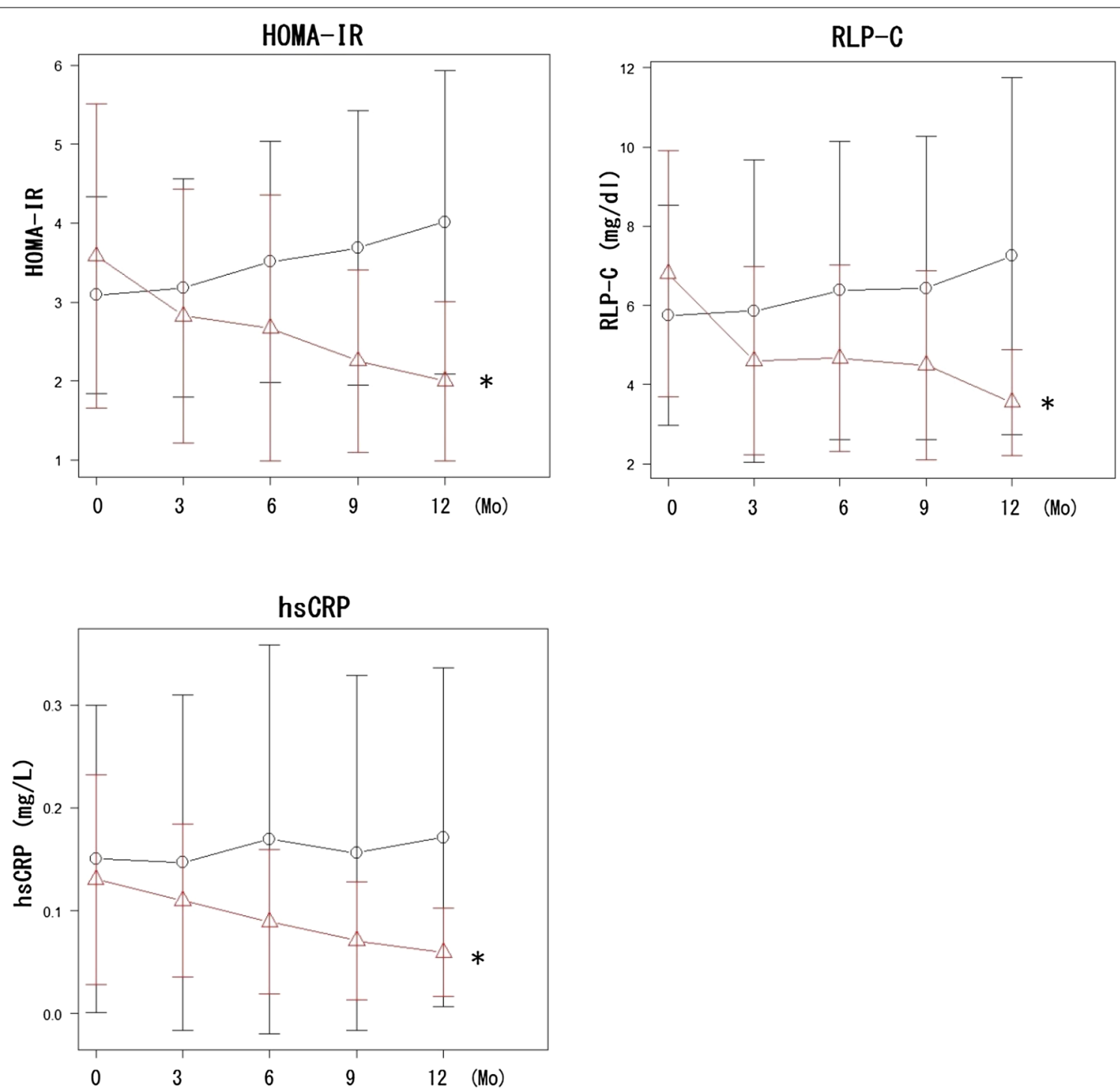

Fig. 1 Time course of HOMA-IR, RLP-C and log[hsCRP] in patients treated with EMPA (triangles, red line) or placebo (circles, black line). Data are presented as mean \pm SD. Group differences parameters between baseline and 12 months later between placebo and EMPA were analyzed ${ }^{*} \mathrm{p}<0.05$ (repeated measures ANOVA); $p=0.0163, p=0.029$ and $p=0.00706$ for HOMA-IR, RLP-C and hsCRP, respectively

\section{Conclusion}

This study showed that the SGLT2 inhibitor, EMPA, ameliorated insulin resistance and might consequently decrease levels of hsCRP and remnant lipoproteins. We also showed that EMPA significantly decreased SBP and DBP. These results suggest that the CV benefits of
EMPA might be driven at least in part, by anti-inflammatory effects that are particularly beneficial for patients with insulin resistance who might have cardiac dysfunction and/or vascular inflammation. This mechanism should cooperate with other EMPA-induced changes such as reduced blood pressure as shown 
Table 3 Association between changes in log[hsCRP] and various parameters coefficients

\begin{tabular}{lclcl}
\hline & Estimate & Std. error & t value & $P$ \\
\hline$\triangle \mathrm{ACR}(\mathrm{mg} / \mathrm{gCr})$ & 0.0006272 & 0.0003121 & 2.01 & 0.0697 \\
$\triangle \mathrm{BMI}$ & -0.004537 & 0.01105 & -0.411 & 0.6892 \\
$\triangle \mathrm{DBP}(\mathrm{mmHg})$ & -0.002671 & 0.002109 & -1.266 & 0.2316 \\
$\triangle \mathrm{eGFR}(\mathrm{ml} / \mathrm{min} / 1.73 \mathrm{~m} 2)$ & 0.0002273 & 0.002345 & 0.097 & 0.9246 \\
$\triangle \mathrm{YGTP}(\mathrm{IU} / \mathrm{L})$ & -0.001513 & 0.001401 & -1.08 & 0.3031 \\
$\triangle \mathrm{AST}(\mathrm{IU} / \mathrm{L})$ & 0.003259 & 0.001935 & 1.684 & 0.1203 \\
$\triangle \mathrm{ALT}(\mathrm{IU} / \mathrm{L})$ & -0.003582 & 0.00151 & -2.372 & $0.037^{*}$ \\
$\triangle \mathrm{HbA} \mathrm{C}(\%)$ & 0.04275 & 0.02592 & 1.65 & 0.1273 \\
$\triangle \mathrm{HDL}-\mathrm{C}(\mathrm{mg} / \mathrm{dL})$ & -0.002794 & 0.001159 & -2.411 & $0.0346^{*}$ \\
$\triangle \mathrm{HOMA}-\mathrm{R}$ & 0.06453 & 0.0254 & 2.541 & $0.0274^{*}$ \\
$\triangle \mathrm{LDL}(\mathrm{mg} / \mathrm{dL})$ & 0.0008015 & 0.0008732 & 0.918 & 0.3784 \\
$\triangle \mathrm{RLPC}(\mathrm{mg} / \mathrm{dL})$ & 0.06442 & 0.02548 & 2.528 & $0.0281^{*}$ \\
$\triangle \mathrm{SBP}(\mathrm{mmHg})$ & -0.002882 & 0.00124 & -2.324 & $0.0403^{*}$ \\
$\triangle \mathrm{TG}(\mathrm{mg} / \mathrm{dL})$ & 0.0000005180 .0003293 & 0.002 & 0.9988
\end{tabular}

$\mathrm{R} 2=0.9855, \mathrm{p}$-value $=4.709 \times 10^{-8}$

Multiple linear regression analysis was used to test the association between change in log[hsCRP] and changes in various parameters across 12 months in EMPA group

hs $C R P$ high sensitivity $C$-reactive protein, $A C R$ albumin-to-creatinine ratio, $B M I$ body mass index, eGFR estimated glomerular filtration rate, GTP, gammaglutamyl transpeptidase, AST aspartate aminotransferase, $A L T$ alanine aminotransferase, $\mathrm{HbA1}$ c hemoglobin A1c, HDL-C HDL cholesterol, LDL-C LDL cholesterol, TG triglyceride, $R L P$-C remnant-like particle cholesterol, SBP systolic blood pressure, $D B P$ diastolic blood pressure

${ }^{*} \mathrm{p}<0.05$

herein and enhanced diuresis [17] to achieve the degree of cardioprotection revealed by the EMPA-REG OUTCOME trial.

\begin{abstract}
Abbreviations
ACR: albumin-to-creatinine ratio; ALT: alanine aminotransferase; AST: aspartate aminotransferase; BMI: body mass index; CAD: coronary artery disease; CV: cardiovascular; eGFR: estimated glomerular filtration rate; EMPA: empagliflozin; FBG: fasting blood glucose; HbA1c: hemoglobin A1c; HDL: high-density lipoprotein; HOMA-IR: homeostatic model assessment of insulin resistance; hsCRP: high sensitivity C-reactive protein; IRI: immunoreactive insulin; LDL: Iowdensity lipoprotein; RLP-C: remnant-like particle cholesterol; SGLT2: sodiumglucose cotransporter 2 inhibitor; $ү$ GTP: gamma-glutamyl transpeptidase.
\end{abstract}

\section{Authors' contributions}

This work was basically done by SH herself. The author read and approved the final manuscript.

\section{Acknowledgements}

The author thanks the members of the Tohto Clinic for helping her implement this work plan

\section{Competing interests}

The author declares that she has no competing interests.

\section{Availability of data and materials}

The datasets analyzed during the current study are not publicly available due to some relevant ongoing studies, but may be available from the corresponding author of this article on reasonable request.
Consent for publication

Not applicable.

\section{Ethics approval and consent to participate}

The study was approved by the ethic committee at the Tohto Clinic, and written informed consent was obtained from all participating patients before the initiation of the study.

Funding

This work isn't receiving any funding.

\section{Publisher's Note}

Springer Nature remains neutral with regard to jurisdictional claims in published maps and institutional affiliations.

Received: 26 October 2018 Accepted: 13 December 2018

Published online: 18 December 2018

\section{References}

1. Zinman B, Wanner C, Lachin JM, Fitchett D, Bluhmki E, Hantel S, Mattheus M, Devins T, Johansen OE, Woerle HJ, et al. Empagliflozin, cardiovascular outcomes, and mortality in type 2 diabetes. N Engl J Med. 2015;373:2117-28. https://doi.org/10.1056/NEJMoa1504720.

2. Kaku K, Lee J, Mattheus M, Kaspers S, George J, Woerle HJ, EMPA-REG OUTCOME ${ }^{\circledR}$ Investigators. Empagliflozin and cardiovascular outcomes in asian patients with type 2 diabetes and established cardiovascular disease-results from EMPA-REG OUTCOME ${ }^{\circledR}$. Circ J. 2017;81:227-34. https ://doi.org/10.1253/circj.cj-16-1148

3. Ridker PM, Everett BM, Thuren T, MacFadyen JG, Chang WH, Ballantyne C, Fonseca F, Nicolau J, Koenig W, Anker SD, et al. Antiinflammatory therapy with canakinumab for atherosclerotic disease. N Engl J Med. 2017;377:1119-31. https://doi.org/10.1056/NEJMoa1707914.

4. Ridker PM, MacFadyen JG, Everett BM, Libby P, Thuren T, Glynn RJ, CANTOS Trial Group. Relationship of C-reactive protein reduction to cardiovascular event reduction following treatment with canakinumab: a secondary analysis from the CANTOS randomised controlled trial. Lancet. 2018;391:319-28. https://doi.org/10.1016/s0140-6736(17)32814-3.

5. Ojima A, Matsui T, Nishino Y, Nakamura N, Yamagishi S. Empagliflozin, an inhibitor of sodium-glucose cotransporter 2 exerts anti-inflammatory and antifibrotic effects on experimental diabetic nephropathy partly by suppressing AGEs-receptor axis. Horm Metab Res. 2015;47:686-92. https ://doi.org/10.1055/s-0034-1395609.

6. Steven S, Oelze M, Hanf A, Kröller-Schön S, Kashani F, Roohani S, Welschof P. Kopp M, Gödtel-Armbrust U, Xia N, et al. The SGLT2 inhibitor empagliflozin improves the primary diabetic complications in ZDF rats. Redox Biol. 2017;13:370-85. https://doi.org/10.1016/j.redox.2017.06.009.

7. Kanda Y. Investigation of the freely available easy-to-use software 'EZR' for medical statistics. Bone Marrow Transplant. 2013;48:452-8. https://doi. org/10.1038/bmt.2012.244

8. Ginsberg HN. Insulin resistance and cardiovascular disease. J Clin Invest. 2000;106:453-8.

9. Merovci A, Solis-Herrera C, Daniele G, Eldor R, Fiorentino TV, Tripathy D, Xiong J, Perez Z, Norton L, Abdul-Ghani MA, et al. Dapagliflozin improves muscle insulin sensitivity but enhances endogenous glucose production. J Clin Invest. 2014;124:509-14. https://doi.org/10.1172/JCI70704.

10. Matsuba R, Matsuba I, Shimokawa M, Nagai Y, Tanaka Y. Tofogliflozin decreases body fat mass and improves peripheral insulin resistance. Diabetes Obes Metab. 2018;20:1311-5. https://doi.org/10.1111/dom.13211.

11. Uemura H, Katsuura-Kamano S, Yamaguchi M, Bahari T, Ishizu M, Fujioka M, Arisawa K. Relationships of serum high-sensitivity C-reactive protein and body size with insulin resistance in a Japanese cohort. PLOS ONE. 2017;12:e0178672. https://doi.org/10.1371/journal.pone.0178672.

12. Effoe VS, Correa A, Chen H, Lacy ME, Bertoni AG. High-sensitivity C-reactive protein is associated with incident type 2 diabetes among african americans: the Jackson Heart Study. Diabetes Care. 2015;38:1694-700. https://doi.org/10.2337/dc15-0221.

13. Alemzadeh R, Kichler J. Gender differences in the association of insulin resistance and high-sensitivity c-reactive protein in obese 
adolescents. J Diabetes Metab Disord. 2014;13:35. https://doi. org/10.1186/2251-6581-13-35.

14. Fukushima H, Sugiyama S, Honda O, Koide S, Nakamura S, Sakamoto T, Yoshimura M, Ogawa H, Fujioka D, Kugiyama K. Prognostic value of remnant-like lipoprotein particle levels in patients with coronary artery disease and type II diabetes mellitus. J Am Coll Cardiol. 2004;43:2219-24.

15. Hattori S. Empagliflozin decreases remnant-like particle cholesterol in type 2 diabetes patients with insulin resistance. J Diabetes Investig. 2018;9:870-4. https://doi.org/10.1111/jdi.12781.
16. Arima H, Kubo M, Yonemoto K, Doi Y, Ninomiya T, Tanizaki Y, Hata J, Matsumura K, lida M, Kiyohara Y. High-sensitivity C-reactive protein and coronary heart disease in a general population of Japanese: the Hisayama study. Arterioscler Thromb Vasc Biol. 2008;28:1385-91. https://doi. org/10.1161/atvbaha.107.157164.

17. McMurray J. EMPA-REG-the "diuretic hypothesis". J Diabetes Complications. 2016;30:3-4. https://doi.org/10.1016/j.jdiacomp.2015.10.012. 